A puntes del CENES

ISSN 0120-3053

Volumen 30 - №. 52

Segundo Semestre 2011

Págs. 9 - 36

\title{
Intercambio y mercado en el pensamiento crítico de Pierre Bourdieu
}

\section{Interchange and market in the critical thought of Pierre Bourdieu}

John Trujillo Trujillo* Nelson Álvarez Marín**

Fecha de recepción: 28 de marzo de 2011

F echa de aprobación: 30 mayo de 2011

* Antropólogo y Magister en Antropología Universidad Nacional, docente-investigador Universidad Central, sede norte, Facultad de Ciencias Administrativas, Económicas y Contables, Departamento de Mercadología. Correo electrónico:jtrujillot@ucentral.edu.co

** Magister en Relaciones Internacionales Universidad Javeriana, Especialista en Gerencia de Marketing Universidad Central, Administrador de Empresas Universidad Externado de Colombia, docente-investigador Universidad Central, sede norte, Facultad de Ciencias Administrativas, Económicas y Contables, Departamento de Mercadología. Correo electrónico: nalvarezm@ucentral.edu.co 


\section{Resumen}

En este artículo se recogen los aportes a la teoría del intercambio y del mercado de Pierre Bourdieu y de algunos especialistas interesados en el trabajo del sociólogo francés. Se aborda así la mirada de Bourdieu como pensador crítico y comprometido social y políticamente, que cuestionó la lógica de las estructuras del intercambio y del mercado en la sociedad y economía occidental y mostró la manera como se han convertido en unidades omnipresentes en la vida social y cultural global. Su trabajo permite comprender el entramado de las relaciones en que se suspenden las dinámicas del intercambio y del mercado en la economía capitalista.

Palabras clave: Pierre Bourdieu, justicia, inequidad, mercadología, sistema capitalista, economía política, sociología económica, antropología económica.

Clasificación JEL: D63, M 31, P1, P16, Z13

\section{Abstract}

This article contains the contributions to the theory of interchange and market of Pierre Bourdieu and also of some specialists concerned in the work of the French sociologist. The text approaches the point of view of Bordieu as a critical thinker that is social and politically engaged, who sought to question the logic of the structures of interchange and the market in Western society, and revealed how they have become omnipresent units in the global culture life. His work allows us to understand the lattice of relations in which the dynamics of trade and market in the capitalist system are suspended.

Keywords: Pierre Bourdieu, justice, inequality, academic marketing, capitalist system, political economy, economic sociology, economic anthropology.

JEL Classification: D63, M 31, P1, P16, Z13 


\section{Introducción}

Este trabajo ha sido adelantado por integrantes del grupo de investigación clasificado en Colciencias, Mercado, Intercambio, Cultura y Organizaciones, quienes participan en el desarrollo de la línea de investigación Dinámicas contemporáneas de los mercados y de las relaciones de intercambio, adscrita al Departamento de M ercadología de la Universidad Central. El estudio es una fase avanzada que busca profundizar en el conocimiento sobre la producción intelectual existente acerca de las teorías del intercambio y del mercado, ámbitos centrales para la consolidación del campo de estudio de la mercadología ${ }^{1}$. En esta fase actual, el grupo tomó la obra del sociólogo francés Pierre Bourdieu (1996) quien trata temas como el intercambio y el mercado en el marco de una lectura sobre las prácticas sociales, que para él se convierten en el escenario para superar -desde el punto de vista teórico y metodológico- las diferencias entre lo objetivo y lo subjetivo o entre el conocimiento fenomenológico y el conocimiento estructuralista en los sentidos que diera el autor a estos enfoques. De allí que la formulación de Bourdieu se encamine a encontrar una nueva época para las ciencias sociales en relación con el debate objetivista-subjetivista, pues consideró como inútil para el estudio de la sociedad evadir cualquiera de las dos posiciones.

\footnotetext{
En fases previas, el equipo de investigación ha revisado distintos enfoques conceptuales nacidos de disciplinas como la antropología, la economía, la sociología, la historia, la administración de empresas y el marketing; y al mismo tiempo ha tratado de comprender los aportes de autores como Karl Polanyi, Marcel Mauss, Arjun Appadurai, George Homans y Karl Marx.
} 
En este punto es necesario advertir al lector especializado en asuntos económicos, que la mirada de Bourdieu y de este artículo no se centra en aspectos particulares de la microeconomía interesada en estudiar los comportamientos económicos de agentes individuales o los usos de los recursos en los niveles de lo microsocial; sino que es una perspectiva sobre los planos de los consumos en el orden de lo social tomado como totalidad. De esta manera busca comprender las implicaciones sociales de los consumos que responden a una lógica económica que se replica en cada agente económico y, por tanto, abarca a toda la sociedad y no a los agentes particulares como tales.

A cerca de Pierre-Félix Bourdieu² es necesario decir que se ha tratado de un destacado sociólogo y filósofo francés de la segunda mitad del siglo 20. R eed-Danahay, 2002; Pinto, 2002; V ásquez, 2002; Téllez, 2002; De La Durantaye, 2004; Swartz, (2003). Swartz (2002) indica que Bourdieu desarrolló una prolífica obra compuesta por unos cuarenta libros y unos quinientos artículos, siendo al tiempo el pensador social más citado desde la década del 90.
Para Bourdieu fue indispensable estudiar el mundo en relación con la posibilidad sociológica de objetivar ciertos aspectos de la realidad. En este sentido se acercó a la óptica durkhemiana donde se ha tratado a la sociedad a partir de hechos - Ios hechos sociológicos-, ejercicio que permite al investigador formularse una aproximación posible para tratar aquello que despierta su interés analítico (Bourdieu, Chamboredon, Passeron, 2002). Sin embargo, Bourdieu entendió que esta perspectiva era insuficiente para estudiar la vida social, y por ello indicó que se hacía también necesario comprender que el conocimiento sobre las acciones de los otros se inscribe en un proceso cognitivo -la subjetividad(Clarke, 2000), en el que las visiones particulares se relacionan con ámbitos basados en posibilidades -estimación de probabilidades- que existen en relación con las situaciones sociales en las que se espera actuar. En este sentido, su trabajo se acerca a la sociología comprensiva de $\mathrm{M}$ ax W eber ( $\mathrm{V}$ ásquez, 2002).

Bourdieu abordó así los campos de la sociología de la cultura (1973), la educación (1998c y 1997a) y de los estilos de vida (1988), tanto desde el

\footnotetext{
2 Pierre Bourdieu nació en 1930, estudió en el Liceo de Pau, en la École Normale Superieure y en La Sorbona cursó Filosofía. Fue profesor en el Instituto de Moulins (Allier), y trabajó en Argelia, París y Lílle. Igualmente fue profesor en la École Normale Superieure, catedrático en el College de France y director de la L'École Pratique de Hauts Études. Ocupó el cargo de director del Centro de Sociología Europea; también dirigió la Escuela Superior de Ciencias Sociales y la revista Actes de la Recherche en Sciences Sociales. Recibió el título de Doctor "Honoris causa" de las universidades Libre de Berlín y Johann-Wolfgang-Goethe de Francfort. Murió en 2002. Swartz (2002) remite a una página web donde aparece una amplia lista de las obras de Bourdieu, y que aquí se sugiere enlazar a través de la dirección: http:/ /www.iwp.jku.at/lxe/sektktf/bb/hyperbourdieustart.html y en http://www.infoamerica.org/teoria/bourdieu1.htm.
} 
plano de la teoría como de la sociología empírica. En ese ejercicio manejó dos conceptos: como pilares fundamentales dos conceptos como fueron la categoría de habitus (Everett, 2002; A twill, 2006) o principio generador visto tanto como forma que crea prácticas como maneras de proceder, pensar y sentir por parte de las personas quienes se ven demarcadas por el entorno de las estructuras sociales que las contienen. Y la categoría de campo (Everett, 2002) que lo refirió como un sistema de redes de relaciones objetivas definidas que se imponen a sus integrantes de acuerdo con su situación, situs, existente y potencial, en función de la disposición de la distribución de las distintas formas de capital o de poder, que, dependiendo de su posición en el juego del campo en la estructura social-, persiguen beneficios particulares (Téllez, 2002; Rehbein, 2007) ${ }^{3}$.

Es importante retomar de la postura de Bourdieu su respuesta al ser interrogado acerca de cómo caracterizar su trabajo, a lo que apuntó que se trataría de un constructivist-structuraism o de un structuralist-constructuvis. Así asumió su comprensión estructuralista, pero en una dimensión diferente a la dada en su momento por autores como Saussure o Lévi-strauss. Al respecto indicó:

Por estructural ismo o estructuralista, quiero decir que existen en el mundo social mismo, y no solamente en los sistemas simbólicos, lenguaje, mito, etc., estructuras objetivas, independientes de la conciencia y la voluntad de los agentes, que son capaces de orientar o de coaccionar sus prácticas - sus representaciones. Por constructivismo, quiero decir que hay una génesis social de una parte de los esquemas de percepción, de pensamiento y de acción que son constitutivos de lo que llamo habitus, y por otra parte estructuras, y en particular de lo que llamo campos y grupo, especialmente de lo que se Ilama generalmente "clases sociales" (Bourdieu, 1996, p. 127-128).

De lo anterior se colige que si bien Bourdieu tomó distancia del estructuralismo, nunca desconoció el aporte de dicha escuela a las ciencias sociales, pues consideró que por primera vez se logró que una ciencia social -la antropología- se impusiera como una disciplina respetable (Butler, 1997). Pero su interés lo llevó, como le sucedió a varios estructuralistas destacados

\footnotetext{
3 La noción de campo, como muchas otras de empleo actual en las ciencias sociales, se originó inicialmente en el mundo de las ciencias físico-naturales. Etimológicamente procede del latín campus que significa "terreno plano". Para el caso particular de la física, se ha dado en llamar campo a una representación que permite esquematizar y trabajar sobre la distribución espacial de magnitudes físicas variables que suelen tener interacción con otras magnitudes físicas. Al respecto Alonso, Caballero y Moreira anotan: "El concepto de campo es fundamental en Física por cruzar ampliosdominios de la disciplina. Se ancla en el concepto matemático de función y se usa para describir el comportamiento de toda magnitud física definida encada punto de una región del espacio y del tiempo, es decir, un camporepresenta una cantidad medible y variable que depende de dónde y cuándo se haya hecho la medida" (Llancaqueo, Caballero \& Moreira, 2003, p. 228). Al respecto no tenemos información si hay relación entre el uso que hace Bourdieu del concepto y su origen en la física en el siglo XIX.
} 
-F oucault, L acan, Barthes- a imaginar cómo romper con las limitaciones de tal propuesta, por lo que tomó distancia de los presupuestos fundamentales del estructuralismo, toda vez que pensaba en el mundo social como: "un espacio de relaciones objetivas trascendente con relación a los agentes e irreductible a las interacciones entre los individuos" (Bourdieu, 1996, p. 21), enunciado en el que se destaca su referencia a negar la existencia de sujetos sociales, al tiempo que privilegia la noción de agentes sociales.

En esta perspectiva, las personas son reconocidas como individualidades capaces no solamente de obedecer y ejecutar ciertas reglas culturales, sino como personas definidas por disposiciones nacidas de sus experiencias; por tanto son seres que actuarán no de forma mecánica sino desde una comprensión de aquello que hacen y del grado en que sus acciones se convierten en favorables para sí mismas, eso sí en tanto respondan a las expectativas generales. De este modo se aparta Bourdieu de la lógica estructuralista en que priman respuestas concretas ante las acciones cotidianas, las cuales se ajustan desde lo dispuesto en las estructuras sociales.

\section{Concepción sobre el intercambio en Bourdieu}

Para comprender la perspectiva de Pierre Bourdieu sobre el tema del mercado y su relación con el intercambio, es indispensable comenzar por indicar que esta posibilidad se construye a través del manejo que él hizo de la categoría de habitus, posiblemente la categoría que más articula sus pensamientos y formulaciones, y que, como lo menciona Téllez, puede definirse así:

Concepto clave de la teoría bourdieusiana, mediante el cual se supera la oposición entre objetivismo y subjetivismo, al permitir la articulación entre las estructuras internas de la subjetividad, y las estructuras y condiciones sociales externas. El habitus es el sistema de disposiciones adquiridas, permanentes y transferibles que permiten actuar, percibir, sentir y pensar de una cierta manera. Estas disposiciones son incorporadas 0 interiorizadas por los agentes sociales en el curso de su vida, a partir del trabajo pedagógico y educativo multiforme y prolongado (socialización, inculcación-apropiación). En resumen, el habitus es un 'operador de cálculo inconsciente', que nos permite orientarnos en un espacio social sin necesidad de reflexión (Téllez, 2002, p. 209-210).

A hora analizamos en la relación de este eje y el asunto del intercambio social.

\subsection{Acerca del intercambio en Pierre Bourdieu}

Desde los intereses del tema que tratamos - las rel aciones entre los sujetos sociales e intercambio y mercado en la 
perspectiva de Pierre Bourdieu- su visión se corresponde con una explicación sobre la condición de los aprendizajes sociales que sirven al sujeto para actuar en ese espacio abstracto Ilamado mercado y para establecer allí relaciones de intercambio, relaciones que vale la pena revisar en términos de lo que Bourdieu Ilamara: “... la experiencia ordinaria de lo social" (1996, p. 18). A sunto que observó en términos de las acciones de las personas, en las que el observador busca: "comprender, a través de mis análisis de la conciencia temporal, las condiciones de la adquisición del 'habitus' económico 'capital ista' en personas formadas en un cosmos precapital ista" (Bourdieu, 1996, p. 21).

Para Bourdieu hay por lo tanto acciones de intercambio en el mercado precapitalista que se vinculan con el mercado capitalista, las que analizó al adelantar y revisar trabajos etnográficos, desarrollando una perspectiva etnológica 0 de interpretación cultural, en la que privilegió conceptos como el de estrategia antes que el de regla (Goodman, 2003; Reed-D anahay, 2004). Observó entonces que en sociedades simples existían estrategias de matrimonio y no reglas de parentesco, tal como lo formuló Lévi-Strauss. Estrategias que se basaban, ante todo en un juego de intercambios, no de orden económico como se comprende hoy, sino con relación a las disposiciones de los grupos sociales para llevar a cabo acciones basadas tanto en las formas aceptadas culturalmente, como en las posibilidades surgidas de las cambiantes situaciones existentes, y que pueden demandar un tipo de relación particular en cada momento. Es decir cada situación de intercambio para movilizar el matrimonio, al ser particular, exigirá de los agentes interesados el hallar la forma de que mediante al guna estrategia, logren concretar la relación que eventualmente puede aparecer como irrealizable por alguna regla del grupo (sobre el tema de las reglas en Bourdieu ver Rehbein, 2007). Bourdieu aclara al respecto que aun cuando el agente no es una simple marioneta dentro de esa 'estructura o modelo social', ello no hace a los agentes "cal culadores racionales", pues para él: "Ias conductas pueden ser orientadas con relación a fines sin estar conscientemente dirigidas hacia esos fines, dirigidas por esos fines" (1996, p. 22), asunto que él resuelve mediante su formulación particular de la noción de habitus.

Para Bourdieu entender el tema del intercambio y del mercado se sitúa, por tanto, en el mismo nivel de preocupación teórica que la comprensión de otros fenómenos sociales, por ejemplo el tema de la dominación masculina, (Chodos\& Curtis, 2002), pues considera que, en términos generales, se debe rebasar la dificultad clásica de si priorizar la perspectiva del sujeto -intelectualista- 0 la de la estructura -mecanicista- para poder comprender la relación entre el agente y el mundo en que se inscribe, que es finalmente la cuestión central que 
interesa a la ciencia social y al pensamiento y filosofía social. Por ello comenzó por tomar como eje de toda mirada de las acciones de los agentes, al modo como se da un ajuste en las disposiciones a la posición: "de las esperanzas a las posibilidades" (Bourdieu, 1996, p. 23), donde las determinaciones se ajustan con relación a las situaciones no mecánicamente ni conscientemente basadas en el conocimiento último de las condiciones objetivas sobre las que se moviliza cada situación, sino sobre las situaciones de vida.

Bourdieu señala que para comprender las acciones del intercambio y las relaciones de mercado -así como de los demás ámbitos de la vida social-, lo que se debe asumir es que hay agentes actuantes y no fijos. De este modo indica: " ... Construir la noción de habitus como sistema de esquemas adquiridos que funcionan en estado práctico como categorías de percepción y de apreciación o como principios declasificación al mismo tiempo que como principios organizadores de la acción, era construir al agente social en su verdad de operador práctico de construcción de objetos" (Bourdieu, 1996, p. 26). Lo anterior obedece a que los agentes están inscritos en lo social, por lo tanto no se trata solo de mirar sus acciones, sino de entender lo que Bourdieu define como estructuralismo genético atendiendo a la necesidad de romper con el estructural ismo a secas, ubicándose así en una perspectiva de comprensión dinámica de lo social, donde se advierte que si bien existen estructuras objetivas -no es que estas desaparezcan, pues se hacen concretas mediantela delimitación que dan los campos, que -para nuestro caso son los campos del intercambio y del mercado, existen al tiempo las acciones de los agentes (frente a los límites y críticas de esta propuesta ver Guillory, 1997). A ello hay que sumar la comprensión sobre la génesis de las estructuras mentales de los individuos -estructuras cognitivas-, producto de dos asuntos: 1. La incorporación de las estructuras sociales; y, 2. El análisis de la génesis de esas estructuras, que han estado articuladas tanto al espacio social como a los grupos que en él se distribuyen, y que serían el resultado de las luchas históricas, tomadas como momentos en los que los agentes ocupan una posición-acción (Bourdieu, 1996).

Así pues, para Bourdieu la tarea del sociólogo se debe dirigir a descubrir, frente a las relaciones del intercambio y del mercado y frente a los demás asuntos sociales: "la necesidad, la coacción de las condiciones y de los condicionamientos sociales, hasta el corazón del 'sujeto', bajo la forma de eso que llamo habitus... al revelar el sistema de condiciones sociales que han hecho una manera particular de ser o hacer, así necesitada sin ser por eso necesaria" (1996, p. 27), de este modo se lograrían identificar las categorías de percepción que encierran el pensamiento, o podría decirse, las categorías dentro de las cuales el pensamiento se ajusta para actuar. 
Diagrama №1. C ampo de la mercadología en el mercado capitalista basado en los intercambios que cobran sentido en lo socio cultural

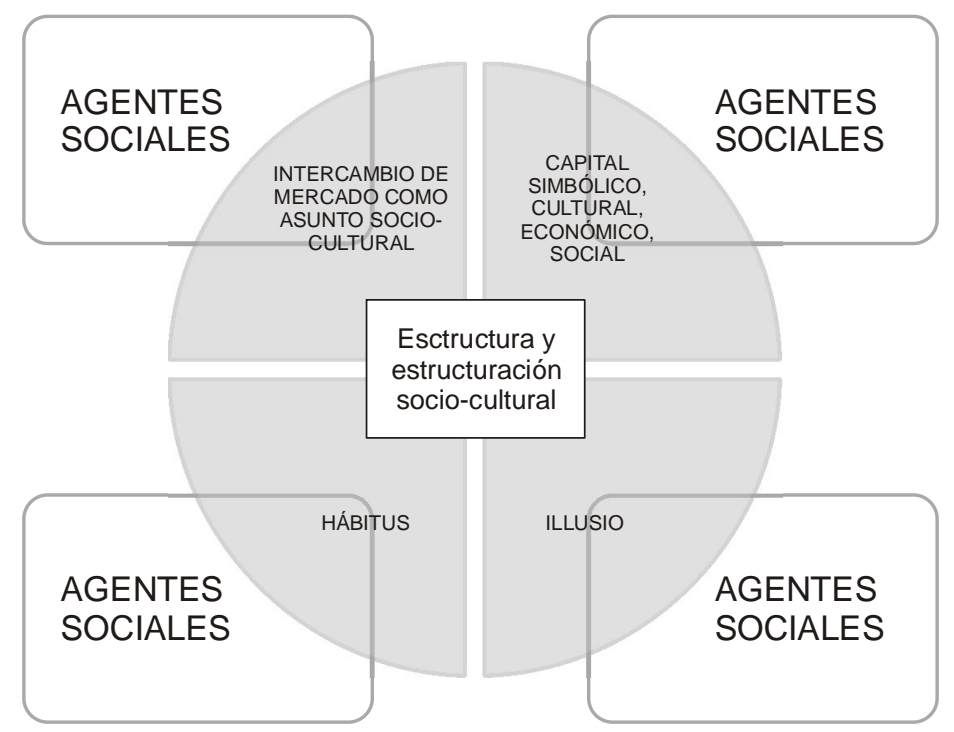

Fuente: los autores.

En un momento simultáneo al ejercicio de teorizar, aparece en Bourdieu la necesidad de realizar investigación empírica que permita, en el propio terreno, verificar lo que conceptualmente se está formulando como un asunto de análisis social, pues se vuelve imperioso ir a las situaciones sociales mismas que se quiere observar, aquellas donde están los agentes de carne y hueso, como él dijera: "los agentes realmente comprometidos, en el mercado, por ejemplo" (Bourdieu, 1996, p. 32), a donde hay que acudir para entender el mundo social a partir de rescatar las experiencias ordinarias de las personas.
A sí puso en juego sus categorías analíticas este pensador, quien se interesó por observar problemas como las luchas por el reconocimiento social, algo que consideró fundamental por ser un asunto dirigido a poner en escena su lógica de la posesión -existenciaparticular de cuatro tipos de capital (Everett, 2002), como son: el social -redes de relaciones-, el simbólico -de conocimiento y reconocimiento- (Hanks, 2005), el económico - de recursos- y el cultural -de clase y de preparación(Glynn, 1993); unido a la mirada sobre la idea de estrategia -no precisamente del todo consciente, calculada 0 
mecánica-, pero presente en la vida social desde la posibilidad de agenciamiento de los sujetos-, y a la idea de la operación de la llamada lógica práctica, presente en las estructuras temporales de los eventos sociales.

Es importante anotar que Bourdieu reconoce que ese discurso construido por el sociólogo es una forma de dominación que nace en el momento en que la disciplina establece una manera de clasificar, que genera diferenciaciones de lo que se reconoce y de lo que se deja de reconocer en su análisis, "de aquello que se incluye, pero particularmente de aquello que se excluye" (Bourdieu, 1996, p. 35).

De esta forma encontramos que se debe comprender la categoría intercambio en el pensamiento de Bourdieu, no como un hecho en sí mismo sino como un hecho social que sólo se entiende cuando se le pone en relación con el campo en que se suceden las relaciones de interacción en que participan agentes sociales, portadores de capitales tanto económicos, como culturales, sociales y simbólicos en el sentido bourdieusiano-, de donde se desprenderá la estrategia de acción dentro del marco estructural de funcionamiento de la cultura, es decir, dentro de sus posibilidades y límites (un ejemplo aplicado al campo de la literatura se ve en Ross, 1997, y en temas de género en Harries, 1997). En este sentido, el asunto del intercambio para Bourdieu se inscribe en el ámbito de la sociología económica, abordado desde la perspectiva de los procesos económicos entendidos en una dimensión simbólica, y no solamente en el orden racional o material (Lebaron, 2004), de tal modo que su preocupación se centra en pensar la naturaleza de los intercambios como hechos sociales y no aislados de tal condición.

Pasamos ahora a apreciar la perspectiva teórica de Bourdieu sobre el mercado.

\subsection{Acerca del mercado en Pierre Bourdieu}

En Bourdieu encontramos una posición que toma al mercado como un lugar de crítica, pues hace referencia a la manera como la ciencia económica lo ha construido en tanto categoría que ha minimizado acciones al ternativas de vida al privilegiarse en su discurso casi una única posibilidad de acogerlo, relacionada con la pretensión dominante de favorecer la idea del libre mercado, avalando de paso la concepción de privilegiar la razón instrumental como medio para 'organizar' el mundo de lo social, marco en el que emergen las diferencias sociales (Lindh $\&$ Dahlin, 2000).

De este modo, para Bourdieu el mercado se lee desde lo que de él ha hecho ese pensamiento dominante de la ciencia económica en su vertiente racionalista, la cual deja en un segundo plano cualquier aspecto que toque los niveles sociológicos, psicológicos o antropológicos, en su afán por asumir preeminentemente al mercado como un 
instrumento para agenciar la política de Ios Estados, especialmente de aquellos que privilegian el libre mercado a ultranza, desde donde se ha ejercido su control a partir de las categorías devenidas de la razón económica.

Esa manera de priorizar las relaciones sociales, que en términos del libre mercado se reducen solo a suponer relaciones económicas, ha generado un completo desconocimiento del tejido social que, por el lado de otros saberes disciplinares, se considera dan respuesta a muchas de las preguntas realizadas sobre el funcionamiento de lo social. En esos terrenos ha existido la preocupación por mirar las acciones de las personas en su cotidianidad, donde se tejen sus vínculos familiares y de grupo local - perspectiva que ha recibido críticas desde al gunos análisis- (Guillory, 1997). Se trata de una suerte de estrategias con las que aquellos seres humanos logran resistir el embate permanente del libre mercado racional consumista, animado por una economía que se ha dedicado a especular desde marcos en apariencia científicos, pero que en el fondo se dirigen tan solo a priorizar las pretensiones del mercado autorregulado, asunto en donde Bourdieu observa que no hay sujeto sino simple mercado de relaciones (Paulson, 1997).

Para Bourdieu, esta dominación que ejerce la ciencia económica sobre el campo económico, hallevado a que otras áreas de la economía y otras disciplinas social es sean vistas como incómodas en el marco de las políticas dominantes de mercado. Así pues, la acción de la sociología se ha convertido, al igual que la de otras áreas disciplinares sociales, en una perspectiva que se toma al margen de los discursos centrales de la modernidad y de la postindustrialización, toda vez que caminan en contravía de los designios de los model os económicos racionalizadores que se ofrecen como posibilidad 'cierta' para hallar el sendero hacia el equilibrio social y la realización de las personas.

Desde aquí, Bourdieu llama la atención sobre las formas subalternas en que deben trabajar la sociología y la antropología el tema del mercado, para lo que propone hacer indagaciones etnográficas como las que realizara en varios espacios el propio pensador francés, en entornos tomados como lugares de referencia donde aparecen al ternativas de solución a la crisis social de cada comunidad, hoy reducidas a los designios de un mundo global que políticamente intenta ser dirigido a la unicidad y a la homogeneidad a través del consumo per se. De allí la importancia que él da a reconocer los mecanismos tradicionales que han coadyuvado a los grupos sociales a afrontar un manejo económico excluyente y poco humanizador.

\section{E I mercado como sistema}

De este modo se reconoce la mirada crítica que tuvo Bourdieu sobre el libre mercado capitalista hoy preponderante. 
L a forma como él se acercó a la explicación sobre la existencia de dicho mercado y la manera como este se aprecia en una perspectiva de sistema, se evidencia en sus trabajos de investigación, en los que con prácticas metódicas estudió las dinámicas mediante las cuales se configuran en la sociedad las relaciones que determinan el lugar que los sujetos ocupan, detallando la cul tura a la que pertenecen.

Para Bourdieu, entonces, el mercado es un campo que se construye de modo diferencial en relación con las pretensiones de esos distintos grupos sociales que componen cada conglomerado humano. A sí se tiene que cada una de las acciones que hacen parte de la vida de las personas está sujeta, en la perspectiva bourdieusiana, a una serie de 'reglas de juego' que determinan sus posibilidades reales y potenciales en el lugar cultural donde cada quien se encuentra inmerso, posición que hoy está como nunca antes determinada por las decisiones políticas y de control que articulan las reglas del mercado y la forma como él opera, casi 'con vida propia'. Deeste modo se da una relación de sujetos sociales que determinan su posición en el entorno del que hacen parte, y en el que posiblemente deberán permanecer si sus condiciones de partida -en términos de capital cultural, económico, social y simbólico- no cambian significativamente desde su nacimiento hasta su muerte.

D os estudios que abordan esta sociología de la vida de los sujetos, se reflejan en
Ios trabajos de Bourdieu: La Distinción (1988) -según Swartz $(2003,2002)$ calificada por la International Sociological A ssociation como la sexta obra más importante producida en el seno de las ciencias sociales durante el siglo XX - y Las estructuras sociales de la economía (2001c). La Distinción, es bien sabido por los especial istas y demás interesados en temas sociológicos, recoge los resultados de una amplia perspectiva investigativa dirigida a detallar cómo se ha llegado a la construcción del gusto en la sociedad -para el caso de ese libro de la sociedad francesa- (Bennett, 2007). Bourdieu desarrolló esta gran labor detallando cómo se han generado niveles diferenciales de acceso al capital cultural, simbólico, económico y social, estrechamente relacionados con las condiciones de partida de vida de cada individuo desde el seno de cada familia. A sí detectó que la construcción del gusto en la sociedad atiende a la generación de una serie de criterios matriciales determinantes para establecer la comprensión cognoscitiva que incide en la definición de la posición que ocupa y ocupará cada quien, a lo largo de los años, en su contexto socio cultural (Loesberg, 1997).

B ourdieu adelanta un ejercicio de campo apoyado en técnicas sociométricas, a lo que se suma la revisión documental extensa sobre las configuraciones de las relaciones sociales atadas a la categoría de clase social, en el sentido de observar cómo se suscitan las acciones de intercambio en términos de saber el modo 
en que varía o no desde la infancia, el lugar ocupado por los sujetos en la sociedad. Ello permite construir una fina telaraña de relaciones que finalmente forjarán las posibilidades estéticas de los sujetos en el lugar donde existen (Paulson, 1997). A partir de allí, como se puede colegir, la diferenciación estética está presente en la mente de cada quien, articulada a la visión del mundo compartida con sus pares sociales, siendo algo que termina volviéndose decisivo para ubicar las posiciones de las personas en el campo de la cultura y de los valores simbólicos con que se representan. E sos espacios serán ocupados en sus sitios de privilegio por aquellos que acopien el bagaje cultural, social, simbólico y económico dominante en la sociedad, al ser promovidos a esos lugares por sus mismos grupos de referencia.

En términos jerárquicos, las " posiciones inferiores" en esa escala de sofisticación estética, irán quedando ocupadas a su vez por las personas establecidas en otros espacios sociales menos favorecidos con respecto a variables como las instituciones donde se estudia o las experiencias de vida cotidiana, las que suelen darse en intensidades y frecuencias diferenciales entre unos grupos sociales y otros, todo en relación con las opciones de cada grupo social para, por ejemplo, ir al teatro, al cine, acceder a la comprensión o producción musical, artística, etc., (Dunn, 1998).

Ese ámbito de opciones se colige de manera directa con las relaciones de intercambio -tanto material como socialpues de forma específica, las posibilidades de acceder a los medios para 'disfrutar' de ciertos ambientes estéticos, se basa en las posibilidades económicas, simbólicas, sociales y culturales de los agentes que buscan participar de esos entornos. De esta manera se requiere contar con un saber simbólico apropiado para actuar en cada espacio, si se espera no portar la sensación de una cierta exclusión que nace del desconocimiento que lo estético cultural -pintura, arte, danza, literatura- suelen demandar en un lugar social o en otro (M oi, 1997; Ross, 1997). A sí se comprende que todo este ejercicio cultural trata de un proceso de intercambio complejo, que se da entre actores que cuentan - 0 no- con las competencias interpretativas que los puedan hacer interlocutores válidos y reconocibles por los otros en entornos específicos de interacción (Dunn, 1998).

En un segundo estudio de Bourdieu, se aprecia una indagación acerca de la forma como evolucionó, a partir de la segunda mitad del siglo veinte, la industria de la construcción de viviendas en Francia y el modo como se ha organizado un mercado de la vivienda relacionado con el proceso político que buscar proveer de techo a las familias que así lo demandasen.

En este ejercicio, Bourdieu se concentró en hacer notar la forma como se crea - literalmente hablando- un mercado de la vivienda, que no surge ni arbitraria ni aisladamente, sino que se constituye 
desde las formulaciones de políticas públicas encaminadas a estructurar un proyecto concreto en esa dirección, proyecto que no dependerá finalmente de las determinaciones gubernamentales sino que estará atravesado por la intervención de las constructoras y los bancos prestamistas, todos agentes interesados en presionar por una suerte de condiciones específicas que darán lugar al origen de un tipo de mercado específico de vivienda, y no a algún otro tipo de mercado distinto al que interesa al mercado abierto.

El mercado de la vivienda es por tanto un espacio artificial donde se mezclan las determinaciones públicas en esta materia, los programas concretos de los gobiernos de turno, las visiones de urbanismo 'vendidas' a la sociedad por las constructoras en relación con la disposición del espacio urbano, a las condiciones promovidas por la banca para 'facilitar' la entrega de préstamos que estimulen la compra de vivienda, a la dinámica publicitaria destinada a 'proyectar' la idea de la compra de vivienda - de cierto tipo de vivienda-, y en menor escala, a los movimientos de los compradoresconsumidores, quienes deberán sopesar sus posibilidades de adquisición de vivienda, camino en el que quedarán los sueños iniciales relegados al plano de las opciones monetarias reales.

En esta perspectiva, Bourdieu revela que en el plano de la dimensión de la planeación social, el proyecto de llevar a la gente a tener una vivienda, se arraiga profundamente en el interés por mantener una estructura de relaciones sociales particulares, vinculada a una política que busca favorecer el establecimiento de hogares unidos especialmente a los intereses dados en la idea de familia. De esta manera se termina respondiendo a la oferta de un mercado que lleva a los compradores a constituir como su proyecto de vida la compra de vivienda y en torno a la vivienda, algo que finalmente será determinante para sus trayectos posteriores al tratarse de una decisión económica que, frente a condiciones de limitado flujo de recursos, termina haciéndose una propuesta de largo plazo y estructurante del futuro de cada persona, pareja y familia.

\subsection{Interés, desinterés e indiferencia en el juego del intercambio: rechazo de la visión reduccionista utilitarista}

Planteamos ahora algunas de las consideraciones y reflexiones que Pierre Bourdieu hiciera con relación al concepto interés, y a la acción interpuesta por los agentes social es en la consecución de sus intereses en función de los intercambios que se suscitan en diversos campos, a través del habitus que media allí.

L a idea principal de Bourdieu en su artículo ¿Es posible un acto desinteresado? $(1988)^{4}$, reside en reconocer que en toda comunidad se constituyen distintos $4 \quad$ El texto es producto de la transcripción de dos clases del College de France pronunciadas en la Facultad de Antropología
y Sociología de la Universidad de Lumiere-Lyon II, en diciembre de 1988. 
espacios sociales basados en sus propias normas y leyes. Así, los agentes dependiendo del campo en donde jueguen, manejarán una disposición o habitus construido sobre su experiencia -especial mente aquella adquirida de forma temprana-, lo que les habilitará en mayor o menor medida para operar en ese escenario a partir del interés que los guíe para estar allí. Según lo mencionó Bourdieu, al gunos de estos campos son el religioso, el artístico, el económico y el literario; y cada uno de ellos involucra un determinado tipo de interés: religioso, artístico, económico, literario o el relativo a cualquier campo particular.

Para entender las operaciones que se suceden en esos campos, Bourdieu introdujo el concepto de interés, y lo diferenció del modo como este ha sido empleado en el campo económico (Swedberg, 2005). Su aproximación al tema se fue construyendo bajo el propósito de probar que la relación práctica entre los agentes sociales y los diversos campos en que interactúan no obedece a un «reduccionismo de visión utilitarista», es decir aquel donde se piensa que los agentes sociales son motivados de manera consciente en función de buscar la máxima eficacia al mínimo coste, algo que los dejaría sujetos a un simple interés económico. Esto es algo que se podría ver representado en relación con los procesos de intercambio, respecto al beneficio monetario 0 al beneficio social esperado por los agentes allí implicados.
De allí que Bourdieu decidiera involucrar en esa mirada sobre la función social del interés, una reflexión acerca del papel de los agentes sociales, desde donde hizo dos preguntas, oportunas aquí para entender el valor de esta propuesta con respecto al funcionamiento de los intercambios en la vida colectiva: ¿por qué resulta hasta cierto punto interesante este término de interés? Y ¿por qué es importante interrogarse sobre el interés que los agentes pueden tener en hacer lo que hacen? (Bourdieu, 1997b, p. 139).

Para abordar el tema, él trabajó esa noción de interés tomado primero como un: "instrumento de ruptura con una visión maravillosa y engañosa de los comportamientos humanos" (Bourdieu, 1997b, p. 139), y lo explicó aduciendo que el interés existe en todos los campos, incluso en el de los intelectuales, pues en todo campo se presentan apuestas interesadas. Su segunda justificación giró alrededor de uno de los postulados de la teoría del conocimiento sociológico, conocido como el principio de la razón suficiente, desde donde, según explicó, se asume que los agentes sociales no hacen cosas por hacerlas, ellos por tanto no son o están locos, pero no por eso se comportan sin razón, simplemente sus acciones son orientadas por razones y no por razonamientos plenos, es decir: "Pueden comportarse de tal modo que, a partir de una valoración racional de las posibilidades de éxito, resulte manifiesto que han tenido razón para hacer lo que han hecho, sin que exista fundamento para 
afirmar que el cálculo racional de las posibilidades haya sido el principio de la elección para lo que han optado..." (Bourdieu, 1997, p. 140). Es por eso que la sociología, dice, considera que en la actuación de los agentes existe una razón en serie, donde se busca articular y dar coherencia a lo que se hace, transformando los actos aparentemente incoherentes y arbitrarios, en una serie coherente que deriva en una interpretación de principio único, siendo en este sentido en que postula: “... que los agentes social es no llevan a cabo actos gratuitos" (Bourdieu, 1997, p. 140).

El acto gratuito, explica Bourdieu, es una idea de lo inmotivado, algo de lo que no sería posible dar razón. Para ejemplificarlo menciona lo que aconteció a $L$ afcadio en la obra de sátira social de A ndré Gide Los sótanos del Vaticano, en donde el personaje mencionado provoca un acto del que no se puede dar razón ${ }^{5}$, se trata de un acto desquiciado, intransigente e irracional frente al cual la ciencia social no tendría otro camino, dice Bourdieu, que desistir sobre una posible interpretación. Esta situación encubre otra en la cual lo gratuito haría referencia a aquello que no vale nada, que no se paga, que no cuesta nada y que no es lucrativo. Revisando ambos sentidos, se entendería que la búsqueda de la razón de ser de un comportamiento puede o no leerse mediante la idea de la persecución de fines económicos; así se comprendería la perspectiva que dio Bourdieu al interés como algo que se puede articular al modo como funcionan los intercambios sociales.

Para profundizar en su marco de trabajo sobre el interés, Bourdieu acogió las categorías de illusio y de líbido, para demostrar que aquellos aspectos son inmanentes a la noción de interés, lo que surgió de observar que los agentes sociales se interesan en las acciones prácticas que involucran el sentido del juego, acciones que se llevan a cabo en distintos universos. Para delimitar la illusio Bourdieu, retomó la idea de Homo ludens del historiador J ohan Huizinga, quien estableció que: “... mediante una falsa etimología, se puede hacer como si illusio, palabra latina que proviene de la raíz ludus (juego), significara estar en el juego, estar metido en él, tomarse el juego en serio" (Huizinga, 2007, p. 141). Huizinga ha aclarado que en latín la expresión lúdica juego, está contenida en la palabra ludus, ludere, que abarca: “... el juego infantil, el recreo, la

5 Lafcadio es un aristócrata, y nos comenta Javier Alcoriza en Dramatispersonae, que en el transcurso de la obra, se dice que altera todos los valores y le imprime un tinte cómico y trágico a la trama en lo que a él respecta. Lafcadio ha entendido que los católicos proceden muchas de las veces con falta de ética; esa situación lo conduce a él a incurrir en una falta de ética, en tanto que su naturaleza es la de un aristócrata, llevándolo a cometer una acción de héroe del acto gratuito. Luego se entiende que la acción gratuita es lo opuesto al proceder de las acciones interesadas o al cálculo que sigue a la conducta supuestamente moral. Se dice en el libro que Lafcadio ha cometido un crimen en donde él procura un acto gratuito, que entorpece la farsa, por lo que debe entregarse a la mañana del día siguiente y de no hacerlo incurriría en el cinismo; pero al hacerlo habrá cruzado el límite de la farsa. Gide deja en un punto muerto, de suspenso su narración, ya que conduce a Lafcadio al extremo de la inconsecuencia. 
competición y la representación litúrgica y también la teatral y también los juegos de azar" (Huizinga, 2007, p. 55). A sí concluyó que el juego es finalmente una cualidad propia e inherente a la forma de vida, cuya finalidad se da: “ ... como una forma de actividad, como una forma llena de sentido y como una función social. No busca los impulsos naturales que condicionarían, de una manera general, el jugar, sino que considera el juego, en sus múltiples formas concretas, como una estructura social" (Huizinga, 2007, p. 15).

U na característica del juego aquí es la idea de libre participación, de allí que el juego no sea una actividad trivial. I gualmente abarca el ser una acción temporera basada en su propia dinámica, que tiene la propiedad de abstracción sobre la vida corriente. El carácter desinteresado del juego y su no pertenencia a la actividad corriente de la vida, la establece Huizinga: “... fuera del proceso de la satisfacción directa de las necesidades y deseos, y hasta interrumpe este proceso. [... ] Se nos presenta el juego en primera instancia como un intermezzo en la vida cotidiana, [... ] pero ya en ésta, su propiedad de diversión regularmente recurrente se convierte en acompañamiento, complemento, parte de la vida misma en general" (H uizinga, 2007, p. 21-22). En esta perspectiva, el juego es algo indispensable de la vida al complementarla, recrearla y tornarla primordial para el individuo, para su bienestar en lo que atañe a lo orgánico y en lo social dado su valor expresivo, espiritual y colectivo que da lugar a una función cultural.

Teniendo en cuenta este punto de vista, Huizinga definió el concepto de juego como:

... una acción u ocupación libre, que se desarrolla dentro de unos límites temporales y espaciales determinados, según las reglas absolutamente obligatorias, aunque libremente aceptadas, acción que tiene un fin en sí mismo y va acompañado de un sentimiento de tensión y de alegría y de la conciencia «de ser de otro modo» que en la vida corriente [... ] el concepto parece adecuado para comprender todo lo que denominamos juego en los animales, en los niños y en los adultos: juegos de fuerza y habilidad, juegos de cálculo y de azar, exhibiciones y representaciones (2007, p. 45-46).

El juego y la illusio en la obra de Bourdieu, a partir de su lectura de Huizinga, asumieron una connotación de fascinación, pues acogió el juego en tanto confabulación entre las disposiciones mentales de quienes lo juegan y las estructuras objetivas del espacio social donde este acontece, algo que se sustentaría en la idea de que cada juego tiene un sentido para sus participantes y por ello vale la pena jugarlo. Pero estar dentro del juego, en la percepción de Bourdieu, causa tensión, es decir, vacilación y genera azar al no saberse cuál será el resultado, 
lo que precisa ir buscando su resolución; de allí la necesidad de esforzarse para obtener el resultado deseado.

Teniendo presente el funcionamiento del juego, Bourdieu retorna al tema del interés poniendo su atención en su opuesto, el desinterés, y en su contraparte la indiferencia, indicando que estaúltima figura alude al agente que está sustraído del juego, algo que ejemplifica mediante la paradoja del asno de Buridán ${ }^{6}$, quien no puede establecer diferencia entre dos posibilidades; así se entiende la distancia que hay entre indiferencia y desinterés. Teniendo esto claro, se llega a la consideración de que en cualquier campo social: económico, artístico, científico, político, etc., aquellos que lleguen a participar, ejercerán un hacer que producirá la acción; aquellos tendrán entonces una relación con la illusio, y por tanto, dice Bourdieu, surgirán en esos campos las relaciones de fuerza, lo que eliminará la posibilidad de que haya agentes indiferentes.

Para Bourdieu, el otro concepto, la líbido, ayuda a dar una explicación sobre la illusio necesaria para que no exista esa indiferencia, ya que en el mundo social la líbido es producto de las pulsiones, contenidas a su vez en la líbido biológica. Por eso, explica, puede haber tantas especies de líbido como campos haya; de ahí que el trabajo de socializar la líbido consistirá en convertir las pulsiones en intereses determinados con respecto a cada campo. Estos intereses específicos interactuarán y cobrarán vida en el espacio social, dice Bourdieu, donde unas cosas se harán importantes y otras indiferentes; así que serán los agentes socializados y constituidos los encargados de establecer las diferencias que se corresponderán objetivamente con las diferencias buscadas en ese espacio. Por ello para Bourdieu surge una diferencia entre quienes nacieron en o dentro del juego y quienes participan de él como conversos, siendo los primeros los mejor adaptados al mismo y por eso los que mayor dominio podrán tener allí al contar con una mejor precisión en sus acciones. En desventaja estarán los agentes que se dirijan a un nuevo terreno - hacia otros campos o disciplinas- donde estarán en presencia de una conversión hacia otro tipo de juego, algo que demandará de ellos un alto esfuerzo para participar adecuadamente en ese nuevo escenario.

Sobre esta base de elementos, Bourdieu rechazó la reducción de la relación práctica de los agentes y de los campos sociales a una visión meramente utilitarista, y se opuso a la idea de que las acciones conscientes de esos agentes se restringen simplemente a la búsqueda del interés económico y al propósito de

6 El asno se enfrenta a una situación absurda en la que, según una de las versiones, no le es posible decidir entre dos montones de heno; ¿cuál consumir primero? puesto que a los dos montones los ve iguales. Entonces el proceder indeciso del asno, entre cuál montón debe comerse primero, lo lleva finalmente a morir de inanición. 
beneficio material en función de un cálculo racional. A sí planteó la pregunta: ¿cómo proceder cuando se pretende reducir esta descripción de la relación práctica entre los agentes y los campos a la visión utilitarista -y la illusio del interés utilitarista-? A lo que respondió:

Para empezar se hace como si los agentes se movieran por razones conscientes, como si plantearan conscientemente los fines de su acción y actuaran para conseguir la máxima eficacia al menor coste. Segunda hipótesis - antropológica-: se reduce todo lo que pueda motivar a los agentes al interés económico, a un beneficio en dinero. Se supone en una palabra que el principio de la acción consiste en el supuesto interés económico, y su finalidad en el beneficio material, planteado conscientemente mediante un cálculo racional (Bourdieu, 1997, p. 144).

Frente a esta formulación, Bourdieu decidió refutar tal acercamiento, y declaró su interés por demostrar, tal como dijo: "... toda mi labor ha consistido en rechazar estas dos reducciones".

Para B ourdieu, (1997, p. 144) a esa idea económica de que los agentes se moverán por razones conscientes, “ ... como si plantearan conscientemente los fines de su acción y actuaran para conseguir la máxima eficacia al menor coste", se contrapone lo que denominó la complicidad que existe de esos agentes y entre el habitus y el campo, es decir, el contexto de sentido donde los agentes y su mundo social viven relacionados en prácticas infraconscientes e infralingüísticas: "¿Acaso un comportamiento humano siempre tiene como fin, es decir como objetivo, el resultado que es el fin, en el sentido del término, de ese comportamiento?", su respuesta obvia es que no, ante lo que indaga: "¿Cuál es pues esta relación tan extraña con el mundo, social o natural, en que los agentes se proponen unos fines sin plantearlos como tales?", a lo que respondió que los agentes sociales están dentro de un juego y que al entender el sentido del mismo, construyen su realidad mediante la percepción de sus estructuras prácticas y sus valores, logrando así el principio de visión y división de sus universos. A sí que él no vio a los agentes sociales como sujetos frente a un problema; por el contrario, los percibió como agentes involucrados en el juego: "... metidos en el juego, en la practicidad, en el quehacer cotidiano, en el presente por venir".

Para completar su perspectiva, Bourdieu tuvo en cuenta los conceptos Husserlianos de P royecto y P rotensión, pues entendió que en el juego existe la perspectiva del futuro en la mente de los agentes involucrados. A cerca de ese factor tomado como proyecto, indicó que este al encontrarse en relación con el futuro del jugador, puede darse o no darse, de allí su importancia.Por otra parte, sobre la noción de protensión 0 
«anticipación preperceptiva» y que en sus palabras significa: "un futuro que no es tal", siendo una particularidad dóxica de lo que es simplemente percibido; de manera tal que esas anticipaciones percibidas son producto de la experiencia, y no vienen incorporadas en un «sujeto puro» sino que se integran a partir de la experiencia que pertenece al habitus como sentido del juego. De esta forma explicó que tener el sentido del juego es llevarlo dentro de sí, luego implica dominarlo, tener un referente histórico y futuro del mismo; de ahí que el buen jugador se caracterizará por saberse anticipar a los acontecimientos del juego, en tanto que el mal jugador siempre estará desfasado, de este modo: “El jugador conocedor del juego tiene tal grado de compenetración que se constituye y forma cuerpo con el juego" (Bourdieu, 1997).

Frente a la segunda reducción racionalista: "... la que consiste en remitirlo todo al interés lucrativo, en reducir los fines de la acción a fines económicos...", explicó que se trataba de una refutación más fácil de establecer que la anterior. Así indicó que el principio de tal error ha estribado en lo que tradicionalmente se ha conocido como el economicismo o hecho de considerar las leyes de funcionamiento de uno de los campos sociales -el campo económico- como válidas para todos los demás campos. De este modo planteó que el fundamento de la teoría de los campos en el orbe social, se caracteriza por una diferenciación de orden progresivo diferente al economicismo.

Para explicarlo acudió a la obra de Durkheim, quien había observado que en las sociedades arcaicas y precapitalista una constitución polisémica y multifuncional en los comportamientos se debía interpretar en conjunto como una totalidad que integraba todos los órdenes: económico, religioso, estético y otros más; este evento, según anotó, no acontece en los actuales sistemas de ideología capitalista, en donde los universos sociales se diferencian entre sí; por ejemplo, la ciencia del arte, de la política o de la religión.

Esa transformación y cambio sociales condujeron, explica Bourdieu, a la aparición de diversos universos 0 campos con leyes propias que los han diferenciado de otros campos; así se explicaría que las leyes fundamentales aparezcan como repeticiones en cada campo. Esta situación la ejemplificó con lo que sucede en el campo económico del que dice, fue creado por los pensadores utilitaristas, cuya norma particular se basó en el principio de: business are business, los negocios son los negocios; o para el caso del arte, expresado por ejemplo, como la escuela del arte por el arte. A sí las cosas, los diversos universos sociales los pensó como regidos por una ley, un nomos que los hace independientes de los demás campos, dándose valor a las acciones propias, siendo autónomos en donde los juicios y principios son irreductibles e independientes frente a los otros universos: " $L 0$ que equivale a olvidar 
que este campo mismo se construyó mediante un proceso de diferenciación, estableciendo[se] que lo económico no es reductible a las leyes que rigen la economía doméstica, a la philia, como decía A ristóteles y a la inversa" (Bourdieu, 1997, p. 149-150).

El pensador francés ejemplifica esas propiedades de diferenciación y «autonomización» con la forma pura y notable del arte por el arte, tendencia que se manifestó en el Renacimiento y abarcó hasta la mitad del siglo XIX, época en la que se rechazaron los propósitos meramente comerciales y mercantilistas aplicados al arte, pues se consideraba que aquellos llevarían a subordinar y condicionar al artista y su producción a exigencias foráneas y a las sanciones producto de esas exigencias. Por el contrario, Bourdieu indicó que ese campo se estableció a partir de la denegación de la economía: "nadie es bienvenido aquí si tiene intereses económicos" (Bourdieu, 1997). Algo similar ocurriría en el campo burocrático, que teniendo como fondo la filosofía hegeliana del Estado, indicaría que el universo burocrático se basa en una premisa rectora: el servicio público, donde los individuos que lo integran deciden sacrificar sus propios intereses por el del interés al servicio público.

Concluyendo frente a la pregunta de Bourdieu ies posible un acto desinteresado? al pensarla desde las implicaciones del intercambio, se observa que su noción de interés nos permite organizar un cuerpo argumentativo, desde donde, creemos, se logra probar y rechazar la idea de que los agentes sociales actúan de forma práctica en los distintos campos 0 universos en donde realizan sus acciones, pues ellos no son solo motivados o interesados desde el orden consciente y racional destinado a la obtención de un beneficio económico, producto de un cál culo. D e esta manera se advierte que la sociedad no está compuesta tan sólo por un universo de nomos de visión reduccionista y utilitarista, sino que se trataría más bien de percibirla en relación con ser constituida por diversidad y multiplicidad de planos sociales que proyectan sus leyes fundamentales en tanto campos a otros universos, poseedores estos a su vez de sus propias leyes fundamentales. De allí la urgencia de limitar la comprensión sobre el papel social de los intercambios al tomarlos como simples ejercicios de interés económico-monetario; siendo más oportuno ubicarlos en el plano del mercado entendido como un campo particular -atravesado por multitud de pequeños campos de intercambio- con lo que se configura como un lugar constituido por sentidos figurados, donde sus participantes juegan basados en su conocimiento y experticia 0 desconocimiento de las reglas que ese campo demanda.

Por ello vemos que el mercado puede abordarse como un terreno meramente económico o uno más interesante de 
índole social, espacio donde los intercambios existirán basados en normas fundamentales y estructuradas en función de su evolución progresiva, algo que les permitirá diferenciarse de otros universos, en especial del campo del mercado exclusivamente económico. D e allí que en campos diferentes a aquel donde prime el sentido utilitarista del juego, aparecerán multiplicidad de actos, por ejemplo aquellos desinteresados en lo económico-monetario, de tal modo que sus jugadores perseguirán un sentido particular, tal como explica Bourdieu, el cual puede guiarse por acciones espontáneas «desinteresadas» para dar alcance a lo buscado conforme a las expectativas generadas por ese juego.

\section{Conclusiones: Bourdieu y la delimitación del campo de estudio del mercado - mercadología}

Desde Bourdieu y su extenso trabajo, se hallan tres grandes betas en relación con los intereses de la mercadología como campo de estudio del mercado, en su interés por ubicar un lugar desde donde esgrimir sus discusiones analíticas y desarrollar su búsqueda en el terreno del conocimiento que le concierne. Estos tres aspectos son: 1. Existencia de un grupo de categorías de análisis sociológico apropiadas para delimitar los intereses del campo de estudio de la mercadología - mercado-, en especial aquella categoría relacionada con la noción de campo. 2. Pertinencia de una mirada de las relaciones de intercambio y del sistema de mercado que se articulan al comprender el asunto como un entramado de conexiones que abarcan tanto elementos simbólicos como aspectos concretos, hoy estructurantes de las relaciones sociales entre las personas y los grupos de interés hegemónicos y no hegemónicos. 3 . F ormulación de una crítica muy seria y fundamentada al modelo neoliberal del libre mercado, desde donde se nos plantea la pregunta por los contenidos éticos y los presupuestos filosóficos que deben acompañar la acción de la práctica profesional y académica en el mercado, teniendo en cuenta que se pretende aportar a la sociedad a partir de generar oportunidades a las personas para que sean seres sociales creativos y no marginales y destinados a vivir en medio de trabajos mecánicos.

Frente al primer aspecto, es útil en relación con la determinación de la noción de campo, reconocer su potencial al permitir a la mercadología pensar en la identificación de los agentes y las dinámicas de mercado que le serían pertinentes al intentar estudiar las interacciones sociales del intercambio. Desde un inicio podría pensarse en la relevancia de incorporar en ese esquema al menos dos niveles de agregados: Primer nivel compuesto por: a. Las políticas en que se basan las decisiones económicas; b. L as decisiones económicas; c. Los intereses de los decisores sobre las políticas económicas; d. El papel de las entidades interesadas en la comercialización o difusión de bienes, servicios o información privada 
o pública; y, e. Los agentes sociales de a pie-ciudadanos-. Y un segundo nivel referido a los condicionantes: $a$. L as motivaciones y deseos de los agentes sociales; b. Los intereses y propósitos conscientes 0 no que movilizan a los agentes sociales; c. Los imaginarios de mundo - cultura- que proveen de sentido lo que hacen los agentes sociales; $y, d$. Los límites económicos, culturales sociales y simbólicos de los agentes sociales para alcanzar sus objetivos.

A partir de esta matriz, se puede imaginar el potencial que reviste la categoría de campo aplicada al mundo de la mercadología que como categoría de anál isis social, unida a la perspectiva de la acción social, permitiría al analista elaborar una visión de trabajo para pensar y plantearse interrogantes en torno al papel de las relaciones de intercambio y del funcionamiento del mercado en la sociedad.

Un segundo nivel de aportes de Bourdieu al campo de la mercadología, emerge desde su comprensión del intercambio como un proceso que cobra sentido no solo en relación con el entendimiento práctico y racional de la toma de decisiones calculadas, sino en el nivel de identificar la existencia de la trama de la vida social del consumo, basada en el nexo entre los significados cultural es y los recursos requeridos para producir significados sociales. Al respecto es oportuno insistir en la necesaria articulación de la mercadología como campo inter y transdisciplinar que debe mantener presente su ineludible articulación con el saber devenido de otras áreas del saber social.

De este modo será insoslayable pasar revista permanentemente a los entronques útiles al análisis mercadológico con respecto a los aportes de, por ejemplo, la psicología experimental con respecto a sus aproximaciones al mundo de la conducta individual; la sociología de grupos en lo que atañe a sus avances en la comprensión de la construcción de la conducta colectiva; la antropología y su preocupación por develar los mecanismos de significación del mundo material vinculado al mundo de lo simbólico; la demografía y su interés por leer el comportamiento de las dinámicas de crecimiento y movilidad social, y ello frente a las condiciones de local ización y deslocalización de las posibilidades laborales y de bienestar material; la cibernética de primer y segundo orden en su pretensión por comprender la relación que se establece entre los flujos de información, la comunicación y la toma de decisiones; la ecología y la ecología humana, y sus preocupaciones frente a los efectos medioambientales generados por cada uno de nosotros y su conexión con los demás seres sociales.

Por último surge el aporte de Bourdieu y su crítica y activismo político militante y continuo en contra del amenazante dominio del modelo neoliberal, que haciendo nido en la dinámica del mercado abierto y sin controles, se ha posicionado a lo largo y ancho del 
planeta a través del agenciamiento intencionado de los organismos multilaterales que introducen sus políticas a nivel local. Así se halla en este pensador una postura de rescate de los valores éticos de la democracia que deben situarse por encima de los valores económicos del capitalismo a ultranza, el cual como modelo de "desarrollo" y "equilibrio social" es inviable, dada la filosofía que lo articula, centrada en la búsqueda del interés personal sobre el colectivo, obstruyendo de este modo las pretensiones de toda democracia real.

A sí las cosas, con Bourdieu el estudioso se adentra en un pensamiento que advierte sobre ese libre mercado que crea continuamente formas hegemónicas de evadir los cuestionamientos, algo que logra al soportarse en la concentración del poder y la riqueza en todo el planeta, sobre la base de un discurso que valida la importancia de la libre economía, quedando así reducida la voz de los sectores contrarios. Desde este otro ángulo, la ciencia económica se ve como una ciencia social que debe enfocarse en la búsqueda de alternativas que favorezcan a la cada vez más marginada y creciente población de un mundo superpoblado; en el que contradictoriamente se advierte sobre los riesgos de la reducción en el descenso de las tasas de natalidad, pues ello puede poner en peligro la sostenibilidad y sustentabilidad del crecimiento económico, el cual finalmente es apropiado por pocos. Este Bourdieu político es uno que no se puede separar de su propuesta académica, pues final mente su proyecto no termina en los libros y auditorios sino que empieza en donde está la gente que se ve marginada de las posibilidades del mudo del consumo, pero que debe responder a la lógica del consumo responsable, sustentable y sostenible.

Por tanto, Bourdieu da a la mercadología no solo elementos analíticos abstractos circunscritos al universo de los expertos y académicos, sino que genera toda una consciencia de la acción de los estudiosos y de las instituciones en relación con su papel y la independencia que deben tener para confrontar los motivos que acompañan hoy las políticas multilaterales sujetas a ideas como la mayor productividad sin detenerse a pensar en la miseria, la pobreza, la falta de oportunidades, la carencia afectiva y la individualización de millones de personas. Ese es finalmente un proyecto que va en contra de la construcción de sociedades y culturas humanizadas, volcándose en una espiral del consumo per se que está llevando al planeta al límite de sus posibilidades ecológicas, y por tanto arriesga la propia existencia de la especie. De esta manera, con Bourdieu se reconoce la importancia de hacer ciencia como medio de conocer y como medio de generar riqueza, pero identificando que sus resultados son políticos, y por eso siempre afectan al mundo; de allí que quienes la realicen deberán,según el autor, asumir una posición pública que sea objeto de seguimiento social. 
En conclusión, esta crítica al mercado capital ista desde Bourdieu no apunta al retorno a un esencialismo o a un natural ismo acorde a la situación de otras épocas-. Su propuesta en concreto sí permite reconocer la relación entre el consumo como hecho inherente a la existencia de las especies en tanto requieren energía para vivir, y el consumismo como apuesta por una sociedad hecha para gastar y no encaminada a realizaciones felicitantes como las que se suelen atribuir a los proyectos democráticos basados en la libertad social, la autonomía y el establecimiento de consensos. El lugar para desarrollar un proceso de esta naturaleza en términos de las acciones mercadológicas se puede iniciar desde la configuración de métodos de articulación entre los planos del consumo social -no individual- y los de la felicidad social, siendo métodos que permitan la concatenación entre el mundo del consumo social y el mundo de la vida social.

Esta propuesta surge del orden de las ideas, de manera que se hace necesario identificar fórmulas para elaborar colectivamente alternativas de consumo que integren en un mismo escenario los requerimientos para fortalecer la vida social y los requerimientos para garantizar un crecimiento económico apropiadamente distribuido. A sí, el análisis de los mercados debe a Bourdieu la propuesta de mantener vigente la crítica al modelo hegemónico capitalista como recurso para hacer perdurar una ética social necesaria para reflexionar sobre lo que se hace desde la planeación social, pero bajo el reconocimiento de los intereses que subyacen a los diferentes agentes y sus capitales culturales, económicos, simbólicos y sociales.

\section{Referencias bibliográficas}

1. Atwill, J. M. (2006). Bodies and Art. Rhetoric Society Quarterly, 36(2), 165170.

2. Bennett, T. (2007). Habitus Clivé: esthetics and politics in the work of Pierre Bourdieu. New Literary $\mathrm{H}$ istory, 38(1), 201-210, 213-228, 239, 5A, $6 \mathrm{~A}$.

3. Bourdieu, P. (1988). La distinción: criterios y bases sociales del gusto. M adrid: Taurus.
4. Bourdieu, P. (1994). Raisons pratiques. Sur la théorie de l'action. París: Seuil.

5. Bourdieu, P. (1996a). Le champ journalistique et la télévision. [Vídeo] Recuperado de http://etnicografica. wordpress. com/2009/08/09/pierrebourdieu-le-champ-journalistique-et-latelevision/

6. Bourdieu, P. (1996b).Sur la télévisón. Suivi de L'Emprise du journalisme, 
Intercambio y mercado en el pensamiento crítico de Pierre Bourdieu John Trujillo Trujillo - N elson Á Ivarez M arín

Liber-Raisons d'agir (editorial fundada por PierreBourdieu). Sobrelatelevisión. (T. K auf, Trad. ). Barcel ona: A nagrama.

7. Bourdieu, P. (1996c). Cosas dichas. Barcelona: Gedisa. (Trabajo original publicado en 1987).

8. Bourdieu, P. (1997ạ). Capital cultural, escuela y espacio social. M éxico: Siglo XXI.

9. Bourdieu, P. (1997b). Razones prácticas: sobre la teoría de la acción. Barcelona: A nagrama.

10. Bourdieu, P. (1998a). Contre-feux. Propos pour servir à la résistance contre l'invasion néo-libérale. París: Raisons d'agir.

11. Bourdieu, P. (1998b). La domination masculine. París: Seuil.

12. Bourdieu, P. (2001a). Contre-feux 2. Pour un mouvement social européen. París: Liber-Raisons d' agir.

13. Bourdieu, P. (2001b). Langage et pouvoir symbolique. París: F ayard.

14. Bourdieu, P. (2001c). Las estructuras sociales de la economía. Buenos A ires: $M$ anantial.

15. Bourdieu, P., Chamboredon, J. C. \& Passeron, J. C. (2002). El oficio del sociólogo. M éxico: Siglo XXI.

16. Bourdieu, P., \& Passeron, J. C. (1973). Los estudiantes y la cultura. Barcelona: Labor.

17. Bourdieu, P., \& Passseron, J. C. (1998c).
La reproducción: elementos para una teoría del sistema de enseñanza. M álaga: Fontamara

18. Butler, M . (1997). The purple turban and the flowering aloe tree: signs of distinction in the early-nineteenth-century novel. M odern Language Q uarterly, 58 (4), 475-495.

19. Clarke, M. (2000). Rosa Parks' performativity, habitus, and ability to play the game. Philosophy today: philosophy in body, culture, and time, 44, 160-168.

20. Chodos, H. \& Curtis, B. (2002). Pierre Bourdieu's masculine domination: a critique. The Canadian Review of Sociology and Anthropology, 39(4), 397412.

21. Dela Durantaye, L. (2004). Biographical illusion and methodological reality. Diacritics, 34 (2), 3-III.

22. Dunn, A. (1998). Who needs a sociology of the aesthetic? Freedom and value in Pierre Bourdieu's Rules of Art. Boundary 2, 25 (1), 87-110.

23. Everett, J. (2002). Organizational research and the praxeology of Pierre Bourdieu. Organizational Research M ethods, 5 (1), 56-80.

24. Glynn, K. (1993). Reading supermarket tabloids as M enippean satire. Communication Studies, 44 (1), 19.

25. Goodman, J. E. (2003). The proverbial Bourdieu: habitus and the politics of representation in the ethnography of K abylia. American Anthropologist, 105 
(4), 782-793.

26. Guillory, J. (1997). Bourdieu's refusal. Modern Language Quarterly, 58 (4), 367-398.

27. Hanks, W. F. (2005). Pierre Bourdieu and the practices of language. Annual Review of Anthropology, 34, 67-83.

28. Harries, E. W. (1997). "Out in left field": Charlotte Smith's prefaces, Bourdieu's categories, and the public sphere. Modern Language Quarterly, 58 (4), 457-473.

29. Huizinga, J. (2007). Homo ludens. M adrid: A lianza.

30. Lebaron, F. (2004). La sociología de Pierre Bourdieu frente a las ciencias económicas. En L. E. Alonso, E. M. Criado, \& J. L. M oreno (Eds). Pierre bourdieu las herramientas del sociólogo. (pp. 131-142). M adrid: Fundamentos.

31. Lindh, G. \& Dahlin, E. A. (2000). Swedish perspective on the importance of Bourdieu's theories for career counseling. J ournal of Employment Counseling, 37 (4), 194-203.

32. Loesberg, J. (1997). Bourdieu's Derrida's K ant: the aesthetics of refusing aesthetics. Modern Language Quarterly, 58 (4), 417-436.

33. Llancaqueo, A., Caballero, M. C. \& Moreira, M. A. (2003). El concepto de campo en el aprendizaje de la física y en la investigación en educación en ciencias. Revista Electrónica de E nseñanza de las Ciencias, 2, (3), 227-253. Recuperado de http://www.saum. uvigo.es/reec/ volumenes/volumen $2 / \mathrm{N}$ umero3/ Art2.pdf

34. Moi, T. (1997). The challenge of the particular case: Bourdieu's sociology of culture and literary criticism. Modern Language Quarterly, 58 (4), 497-508.

35. Reed-Danahay, (2002). D. Remembering Pierre Bourdieu, 1930-2002. Anthropological Quarterly, 75 (2), 375-380.

36. Reed-Danahay, D. (2004). Tristes Paysans: Bourdieu's early ethnography in Bearn and Kabylia. Anthropological Quarterly, 77 (1), 87-106.

37. Rehbein, B. (2007). Configurations of globalization in Laos and Cambodia. International Asian Forum. International Quarterly for Asian Studies, 38 (1/2), 67-85.

38. Paulson, W. (1997). The market on printed goods: on Bourdieu's rules. Modern Language Quarterly, 58 (4), 399-415.

39. Pinto, L. (2002). Pierre Bourdieu y la teoría del mundo social. M éxico: Siglo XXI.

40. Ross, T. (1997). "Pure poetry": cultural capital and the rejection of classicism. Modern Language Quarterly, 58 (4), 437-456.

41. Swartz, D. L. (Diciembre, 2003), In Memoriam Pierre Bourdieu (19302002): drawing inspiration from Bourdieu's sociology of symbolic power. Special issue on The sociology of symbolic power: a special issue in memory of Pierre Bourdieu. Theory and 
Society, 32, (5/6), 519-528.

42. Swartz, D. L. (A gosto, 2002). In M emoriam: Pierre Bourdieu 1930-2002. Theory and Society, 31, (4), 547-553.

43. Swedberg, R. (2005). Can there be a sociological concept of interest? Theory and Society, 34, (4), 359-390.
Recuperado de http://www.jstor.org/ stable/4501729.

44. Téllez, G. (2002). Conceptos básicos y construcción socioeducativa. Bogotá: U niversidad Pedagógica N acional.

45. Vásquez, F . (2002). Pierre B ourdieu: la sociología como crítica de la razón. Barcelona: M ontesinos. 